\title{
OBSERVATIONAL ASPECTS OF WOLF-RAYET ATMOSPHERES
}

\author{
I.I. ANTOKHIN \\ Sternberg State Astronomical Institute \\ Universitetskij pr., 13, Moscow 119899, Russia
}

\begin{abstract}
Observational data suggest that WR atmospheres must be considered as highly inhomogeneous objects incorporating strong shocks induced by radiative instability and full-scale supersonic turbulence, with relatively small mean mass-loss rates.
\end{abstract}

Key words: Wolf-Rayet - spectroscopy - photometry - polarimetry - variable

\section{Introduction}

Great progress in modelling Wolf-Rayet atmospheres has been achieved during the past decade. Schmutz (1991) and Hamann (1994) have reviewed the results of fitting observed spectra by means of a "standard model" of a WR wind and demonstrated that generally good agreement between the theory and observations can be achieved for the continuum distribution as well as for the strengths and profiles of emission lines. At the same time, the understanding is growing that WR atmospheres are more complex objects than is assumed in the "standard model". Since observational data supporting the "standard model" are considered in detail in cited reviews of Schmutz and Hamann, here we focus on: (i) contradictions between observations and the "standard model"; and (ii) observational data, which show that the "standard model" has not been designed to account for e.g., data on intrinsic variability and polarimetric data.

\section{Contradictions between observations and "standard model"}

Problems of the "standard model" have been mentioned by many authors (e.g., Underhill 1991a; Hillier 1991a; Hamann 1994). Some contradictions, such as incorrect metal-line strengths could possibly be due to inadequate atomic data, as well as to some simplifying assumptions of the "standard model" (Hillier 1991a). Others, however, indicate real problems. These are:

(i) Calculated shortward-displaced absorption components in some lines are stronger than observed ones (Hamann et al. 1988; Underhill 1991a). It would appear reasonable that the real column density along the line of sight to the stellar core is smaller than the model one, thus implying $\dot{M}_{\text {real }}<$ $\dot{M}_{\text {model }}$. Alternatively (Hillier 1991a) one can allow for a fluctuating velocity field. So far only one attempt has been undertaken in this direction, by Puls et al. (1993). Low values of $\dot{M}\left(\leq 10^{-6} M_{\odot} y r^{-1}\right)$ for WR stars have 
been postulated by Underhill (1991b) $\left(c f . \dot{M} \sim\left(10^{-5}-10^{-4}\right) M_{\odot} y r^{-1}\right.$ from the "standard model", with a tendency to the higher value). The problem, however, is that in the homogeneous wind model the emission part of a line profile cannot be reproduced with a small $\dot{M}$. Hillier (1991b) has shown that in a clumped model of the WR wind one could reproduce the emission part of line profiles reasonably well with $\dot{M}$ about 2 times smaller than $\dot{M}$ in the "standard model". However, in his 1-D model (in which clumps are represented by spherical shells) the absorption in the He I $\lambda 5876$ line remains strong ( $c f$. his Fig. 16). The absorption might decrease in a more realistic model of clumps localised in space (e.g., in form of "blobs") if the filling coefficient is small (Antokhin et al. 1992).

(ii) The electron scattering wings produced in the "standard model" are stronger than observed ones (Hillier 1991a). Hillier (1991b) has shown that significant reduction in the strength of an electron scattering wing can be achieved in a model of clumped wind for a lower mean mass-loss rate.

(iii) The double-peaked shape of the He I $\lambda 5876$ line (in WR6 and WR134) and flat-topped shape of this line in other strong lined WN and some WC stars are not fully reproduced in the "standard model" (Hamann 1994). Underhill and co-authors (see Underhill \& Yang 1991 and references therein) have suggested that double-peaked line profiles could be explained in a disklike model of the WR wind. They also claimed that systematic shifts of central wavelengths of $\mathrm{He}, \mathrm{N}$ and $\mathrm{C}$ lines indicate ejection of the plasma from and its infall toward the star. For WR134, Vreux et al. (1992) have found that if patterns on the tops of emission lines are used to derive wavelengths of emission lines instead of usual measurements of line "centers", any systematic shifts are absent. Thus, the results of Underhill and co-workers may be an artifact of asymmetric line shapes and/or blends. Antokhin et al. (1992) have suggested that the double-peaked profile of He I $\lambda 5876$ could be explained by local asymmetry of blobs rather than global asymmetry of a wind. However, the problem with any "geometrical" explanation is that if it were true, one would expect similar line-profiles of other He I lines, which is not the case (Vreux et al. 1992). These authors have assumed that the shortward emission peak in He I $\lambda 5876$ is simply a blend. Considering that "standard model" tends to produce a flat-topped profile of the He I $\lambda 5876$ line, its observed shape does not convincingly indicate that WR winds are asymmetric.

(iv) The He I $\lambda 10830$ triplet shows extra emission subpeaks in the otherwise rather flat-topped profiles in many broad-line WR stars (Eenens 1991; Eenens \& Williams 1994). Eenens (1991) has suggested these peaks are a signature of jets. However, the same problem as before persists: other He I lines do not show such peaks.

(v) Black troughs in saturated P-Cygni profiles of resonance lines and accompanying "blue edges" are not reproduced by means of the "standard 


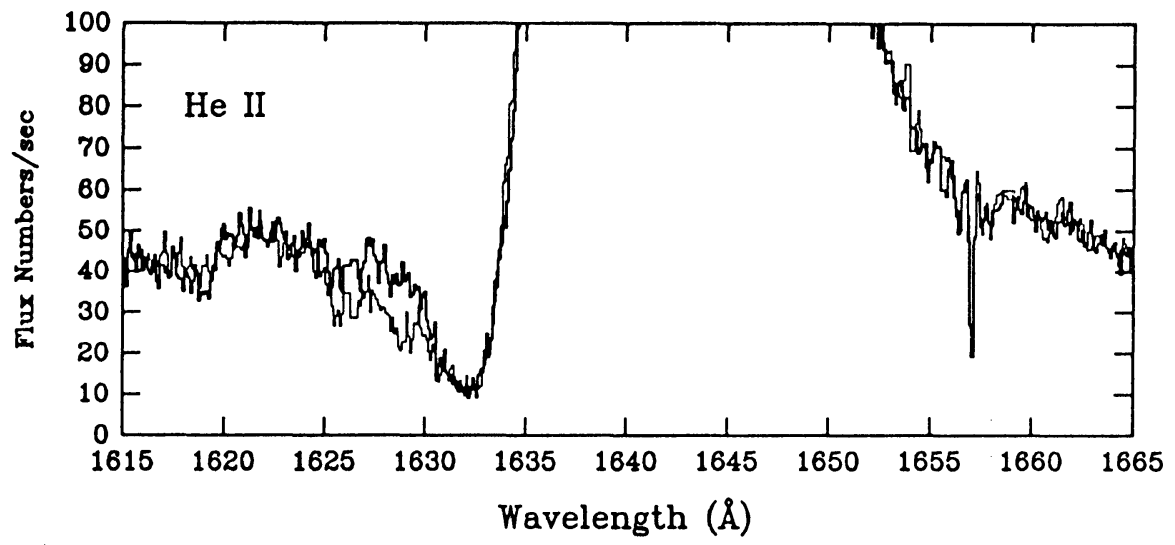

Fig. 1. IUE spectra of WR6 illustrating the variations in some P-Cygni profiles (from St-Louis et al. 1993a)

model" (cf. Hillier 1988). Large turbulent velocities $\left(\Delta V>200 \mathrm{kms}^{-1}\right)$ or acceleration of the wind to velocities higher than $v_{\infty}$ at low electron densities have been mentioned as possible solutions of the problem. Observations of "blue edge" variability (see below) and pilot modelling of Puls et al. (1994) indicate that the latter mechanism is workable through the development of strong shocks in the outer wind.

(vi) The "standard model" is unable to explain an increase of the effective radius of the WR component of the binary WN5+O6 star V444 Cygni in the infrared (Hamann \& Schwarz 1992; Cherepashchuk et al. 1994). Again, a clumpy structure of the WR wind appears to be a reasonable explanation.

\section{Intrinsic variability}

Intrinsic variability itself does not influence the model parameters, because "the deviations from the mean profile are generally not larger than the deviations of the synthetic profiles from the observed (mean) profile" (Schmutz 1991). The conclusion is true, however, only if we believe the assumptions central to the "standard model", i.e., spherical symmetry and homogeneity. The importance of the intrinsic variability lies in the fact that it indicates that these assumptions are not valid. A model based on different assumptions (e.g., clumped wind) might be able to produce a theoretical spectrum similar to the one obtained with the "standard model" but at different values of stellar parameters, as has been indeed demonstrated by the pilot calculations of Hillier (1991b) and Antokhin et al. (1992).

In a previous review of intrinsic variability of WR stars (Moffat \& Robert 1991) the conclusion was reached that the general pattern of variability "can 
be explained by one common phenomenon: stochastic formation, propagation and decay of density enhancements in the winds". New observations complicate this picture.

\section{1 "Blue EDGES" AND Discrete abSOrPtion fEATURES}

Time resolved UV spectroscopy of WR6 (Willis et al. 1989) and WR136 (WN6) (St-Louis et al. 1989) has lead to the conclusion that existence of "soft blue absorption edges" in P-Cygni profiles and their variability are qualitatively consistent with the propagating shock models (Owocki et al. 1988; Puls et al. 1994). The "blob" hypothesis (Moffat \& Robert 1991) seems to be unlikely to account for these data, because an unknown mechanism to accelerate the blobs to super-terminal velocity must be postulated.

Later, an extensive data set consisting of 130 high-resolution IUE spectra over a period of six days for WR6 has been published by St-Louis et al. (1993a) (Fig. 1). The previous conclusion was generally confirmed. No discrete absorption features have been seen during these observations, unlike in O-type stars, due possibly to saturation effects.

In 1992, Prinja and Smith (1992) reported the discovery of blueward migrating discrete absorption features in the unsaturated He II $\lambda 1640 \mathrm{P}$ Cygni profile of WR24 (WN7+abs). Their properties resemble of those in Otype stars, providing additional support for a common physical mechanism (radiative instabilities) for wind variability in $\mathrm{O}$ and WR stars.

\subsection{OPTICAL EMISSION LINE VARIABILITY}

Systematic micro-variability in optical emission lines of some WR stars showing evidence for "bumps" moving away from the center of line, has been reported in the late 1980s (Moffat \& Robert 1991) and interpreted as a signature of blobs moving away from the star. That interpretation has been criticized by Underhill et al. (1990) and Underhill \& Yang (1991).

To (i) quantify the information about subpeak parameters in a more objective way and to (ii) move to small features which could not be recognized "by eyes", Robert (1992), Moffat \& Robert (1992) and Moffat et al. (1993) have applied multi-Gaussian and wavelet analysis technique. The main result is that scaling laws between subpeaks parameters (flux, velocity dispersion, lifetime, position on the line, $c f$. Fig.2) strongly support the hypothesis of anisotropic supersonic compressible turbulence in the wind. Moffat \& Robert (1994) have shown that a turbulent wind with observed properties could lead to a decrease in the derived value of $\dot{M}$ by a factor of $\geq 3$ compared to homogeneous wind of the same free-free or subordinate line flux.

Another conclusion of Robert (1992) is that, at least in some WR stars, acceleration of blobs is very slow $(\beta>10)$. Thus, assuming that blobs are good tracers of the flow velocity the usually adopted value of $\beta \simeq 1$ seems 

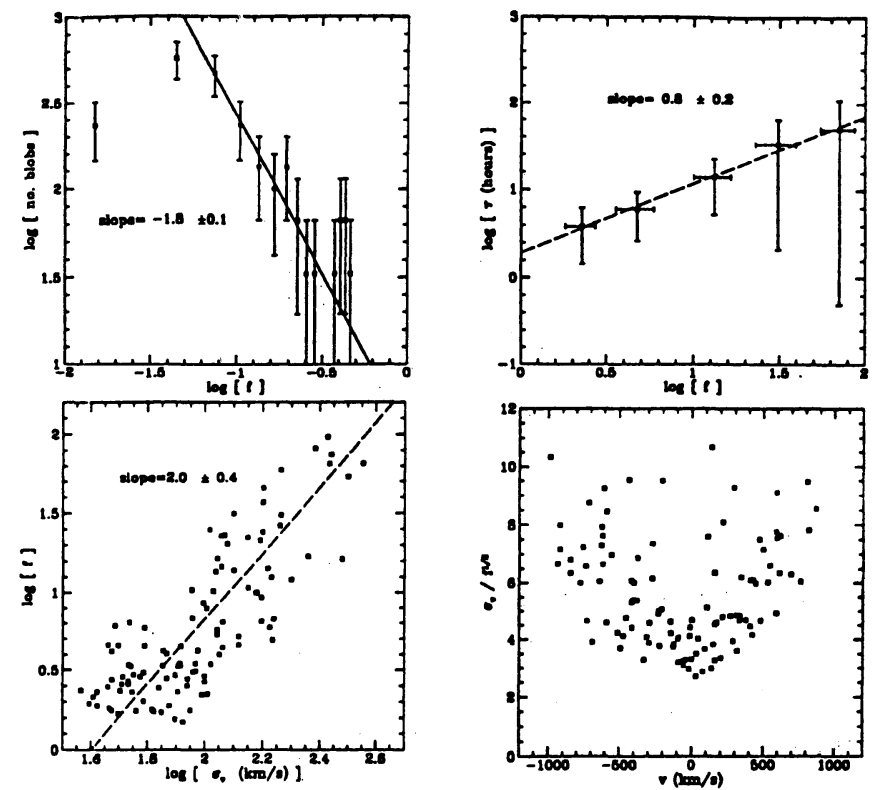

Fig. 2. Results of wavelet analysis for C III $\lambda 5696$ profile of WR135 (WC8) (from Moffat et al. 1993): (a) flux spectrum; (b)flux vs. velocity dispersion; (c)life-time vs. flux; (d)normalized velocity dispersion vs. position on the line.

inadequate. This result is compatible with the conclusion of Koenigsberger (1990) obtained from an analysis of phase-dependent Fe V/Fe VI pseudocontinuum in binary $\mathrm{WR}+\mathrm{O}$ stars.

A further complication of the overall picture comes from observations of WR134 performed by Vreux et al. (1992). The authors have shown that while the pattern of micro-variations of emission line profiles in several nights was similar to that found by Moffat et al., there were also many events which differ remarkably from a picture of "gently moving" blobs. In particularly, there are preferable points (nodes) in the line profiles, where the variations are much smaller than in between. Two typical examples of variability are illustrated in Fig.3: (i) a given pattern repeats itself after some time; (ii) two patterns are nearly the mirror image of one another. To explain all observational facts, the authors suggest a tentative model of a wind resembling the Luminous Magnetic Rotator model (Cassinelli 1991).

\subsection{PhOTOMETRIC VARIABILITy}

A number of photometric investigations of apparently single WR stars have been undertaken since the review of Moffat \& Robert (1991). The results are:

(i) Several stars (WR24 (WN7+abs), WR25 (WN7+abs), WR78 (WN7), WR108 (WN9-Ofpe) (Gosset et al. 1994), WR82 (WN8) (Antokhin et al. 

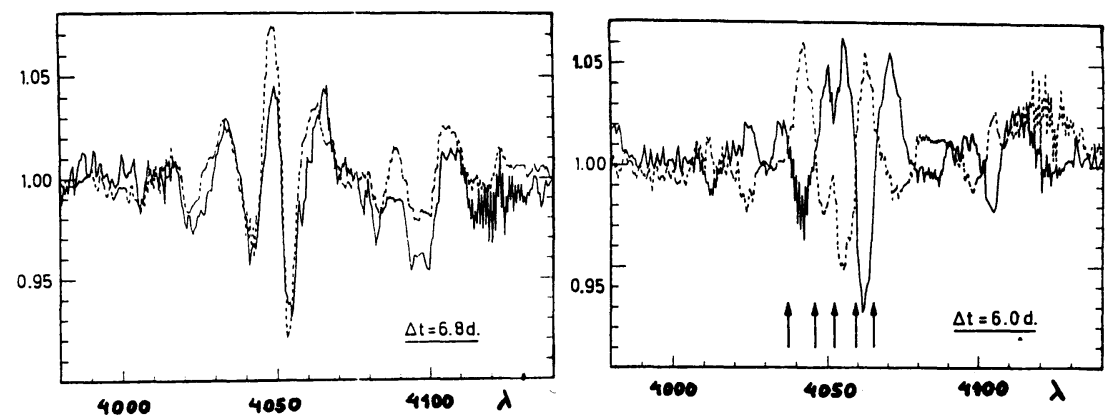

Fig. 3. Examples of the (a) repeatability and (b) "mirror" situation in the N IV $\lambda 4058$ profile of WR134 (from Vreux et al. 1992).

1994b)) show random low-amplitude variability in the continuum, exceeding observational noise.

(ii) The WN8 stars WR16 and WR40 show along with random lightvariations some degree of periodicity (Gosset et al. 1994; Antokhin et al. 1994b). Matthews \& Moffat (1994) have suggested that photometric variations of WR40 may be understood as a signature of orbital and rotational periods in a WR binary system.

(iii) Blecha et al. (1992) have reported the discovery of radial pulsations in WR40 with a period of $\simeq 10$ minutes. This kind of pulsations had been predicted theoretically (Maeder \& Schaller 1991). The result, however, has not been confirmed by subsequent observations (Gosset et al. 1994, Marchenko et al. 1994).

(iv) No periods $\leq 1 \mathrm{~d}$ which could indicate non-radial pulsations (NRP) have been found in all mentioned stars as well as in WR6 (WN5) (Gosset et al. 1994; Antokhin et al. 1994a). However, Antokhin et al. (1994b, see also these proceedings) discovered a 3.5 h periodicity in WR66 (WN8). This may be the first clear example of NRP among WR stars. Another tentative interpretation is that we observe the NS-companion at spiral-in stage.

\section{Polarimetry}

Great progress in polarimetric investigations of WR stars, both single and binary, has been achieved in recent years. Since detailed reviews on polarimetric data can be found in Schulte-Ladbeck (1994), only the main results will be summarized here:

(i) $\sim 80 \%$ of the investigated WR stars show intrinsic polarimetric variability, indicating that their winds are both temporally and spatially variable.

(ii) Line effects (depolarization across a line) are present in about $25 \%$ of the investigated WR stars. Of those only WR6 and WR134 appear to have 
axisymmetric winds.

(iii) Polarimetric estimates of mass-loss rates in binary $\mathrm{WR}+\mathrm{O}$ stars tend to be smaller than those from radio data and from modelling emission-line spectra of WR stars (St-Louis et al. 1993b).

\section{Conclusions}

The observations considered strongly suggest that mass-loss rates in WR atmospheres are smaller than those predicted by the "standard model" (possibly $\left.<10^{-5} M_{\odot} y r^{-1}\right)$. If a star's $\dot{M}$ is small, its wind must contain density enhancements, to produce the observed flux in emission lines. There is also direct observational evidence for the inhomogeneity of WR winds.

The qualitative observational picture of WR winds has become more complicated than suggested in Moffat \& Robert (1991). Instead of randomly ejected blobs, gently moving in an otherwise smooth wind we come to an empirical model which incorporates strong shocks induced by the radiative instability of the wind and full-scale supersonic (anisotropic) turbulence. In some cases, there is evidence for global asymmetry of a WR atmosphere. The usual $\beta$ law is possibly not adequate. The question is how the observational data could be used in future models of WR atmospheres. Most of them could serve as constraints. Scaling laws from recent papers of Robert (1992) and Moffat et al. (1993) could hopefully be directly incorporated into existing models. New time-resolved simultaneous observations in different modes will hopefully reveal new empirical correlations and provide new insights into the physics of WR atmospheres.

\section{Acknowledgements}

This work has been partly supported by the Russian Fund of Fundamental Research through the grant No. 93-02-17113. The author acknowledges the support of a Travel Grant from the International Science Foundation (grant No. 1261/1).

\section{References}

Antokhin, I.I., Nugis, T., Cherepashchuk, A.M. 1992, Sov. Astron. 36, 260

Antokhin, I.I., Bertrand, J.-F., Lamontagne, R., Moffat, A.F.J. 1994a $A J$ 107, 2179

Antokhin, I.I., Bertrand, J.-F., Lamontagne, R., Moffat, A.F.J., Matthews, J.M. 1994b, in preparation

Blecha, A., Schaller, G., Maeder, A. 1992, Nature 360, 320

Cassinelli, J.P. 1991, in: K.A. van der Hucht \& B.Hidayat (eds.), Wolf-Rayet Stars and Interrelations with other Massive Stars in Galaxies, Proc. IAU Symp. No.143 (Dordrecht: Kluwer), p. 289

Cherepashchuk, A.M., Antokhin, I.I., Djapiashvili, E.B., Nadjip, A.E., Shenavrin, V.I. 1994, Astron. Reports, in press 
Eenens, P.R.J. 1991, in: C. Jaschek \& Y. Andrillat (eds), The Infrared Spectral Region of Stars (Cambridge: CUP), p.137

Eenens, P.R.J., Williams, P.M. 1994, MNRAS , in press

Gosset, E., Rauw, G., Manfroid, J., Vreux, J.-M. 1994, in: C. Sterken \& M.J.H. de Groot (eds.), The Impact of Long-Term Monitoring on Variable-Star Research, Proc. NATO Workshop, (Dordrecht: Kluwer), p.101

Hamann, W.-R. 1994, in: D. Vanbeveren, W. van Rensbergen \& C. de Loore (eds.), Evolution of Massive Stars: A Confrontation between Theory and Observations, Sp. Sci. Rev. 66, 237

Hamann, W.-R., Schmutz, W., Wessolowski, U. 1988, $A \mathcal{E} A$ 194, 190

Hamann, W.-R., and Schwarz, E. 1992, $A \mathscr{E} A$ 261, 523

Hillier, D.J. 1988, $A p J$ 327, 822

Hillier, D.J. 1991a, in: K.A. van der Hucht \& B.Hidayat (eds.), Wolf-Rayet Stars and Interrelations with other Massive Stars in Galaxies, Proc. IAU Symp. No.143 (Dordrecht: Kluwer), p. 59

Hillier, D.J., 1991b, $A \& A$ 247, 455

Koenigsberger, G. 1990, $A \mathscr{E} A$ 235, 282

Maeder, A., and Schaller, G. 1991, in: K.A. van der Hucht \& B.Hidayat (eds.), Wolf-Rayet Stars and Interrelations with other Massive Stars in Galaxies, Proc. IAU Symp. No.14.3 (Dordrecht: Kluwer), p. 167

Marchenko, S.V., Antokhin I.I., Bertrand, J.-F., Lamontagne, R., Moffat, A.F.J., Piceno,A., Matthews, J.M. 1994, $A J$,in press

Matthews, J.M., Moffat, A.F.J. 1994, $A \& A$ 283, 493

Moffat, A.F.J., Robert, C. 1991, in: K.A. van der Hucht \& B.Hidayat (eds.), Wolf-Rayet Stars and Interrelations with other Massive Stars in Galaxies, Proc. IAU Symp. No.14.3 (Dordrecht: Kluwer), p. 109

Moffat, A.F.J., Robert, C. 1992, in: L. Drissen, C. Leitherer, A. Nota (eds.), Nonisotropic and Variable Outflows from Stars, ASP Conf. Series 22, p. 203

Moffat, A.F.J., Lépine, S., Henriksen R.N., Robert, C. 1993, ASP Conf. Series in press

Moffat, A.F.J., Robert, C. 1994, ApJ 421, 310

Owocki, S.P., Castor, J.I., Rybicki, G.B. 1988, $A p J$ 335, 914

Prinja, R.K., Smith, L.J., 1992, $A \notin A$ 266, 377

Puls, J., Pauldrach, A.W.A., Kudritzki, R.P., Owocki, S.P., Najarro, F. 1994, Rev. Mod. Astron. 6, in press

Puls, J., Owocki, S.P., Fullerton, A.W. 1994, $A \varepsilon^{\&} A$ in press

Robert, C. 1992, Ph.D. thesis, Univ. de Montréal

Schmutz, W. 1991, in: K.A. van der Hucht \& B.Hidayat (eds.), Wolf-Rayet Stars and Interrelations with other Massive Stars in Galaxies, Proc. IAU Symp. No.14.3 (Dordrecht: Kluwer), p. 39

Schulte-Ladbeck, R.E. 1994, these proceedings

St-Louis, N., Smith, L.J., Stevens, I.R., Willis, A.J., Garmany, C.D., Conti, P.S. 1989, $A \& A$ 226, 249

St-Louis, N., Howarth, I.D., Willis, A.J., Stickland, D.J., Smith, L.J., Conti, P.S., Garmany, C.D. 1993a, $A \& A$ 267, 447

St-Louis, N., Moffat, A.F.J., Lapointe, L., Efimov, Yu.S., Shakhovskoy, N.M., Fox, G.K., Piirola, V. 1993b, ApJ 410, 342

Underhill, A.B. 1991a, ApJ 383, 729

Underhill, A.B. 1991b, in: K.A. van der Hucht \& B.Hidayat (eds.), Wolf-Rayet Stars and Interrelations with other Massive Stars in Galaxies, Proc. IAU Symp. No.143 (Dordrecht: Kluwer), p. 53

Underhill, A.B., Gilroy, K.K., Hill, G.M., Dinshaw, N. 1990, ApJ 351, 666

Underhill, A.B., and Yang, S. 1991, ApJ 368, 588

Vreux, J.-M., Gosset, E., Bohannan, B., Conti, P.S. 1992, A $6 A$ 256, 148

Willis, A.J., Howarth, I.D., Smith, L.J., Garmany, C.D., Conti, P.S. 1989, A $\varepsilon^{A}$ Suppl. 77, 269 


\section{DISCUSSION:}

Moffat: While recent quantitative analyses have advanced our understanding of substructures in WR winds greatly, we are still a long way from understanding their nature, let alone their origin. For example, we only are seeing the tip of the iceberg; we have no (direct) idea of what happens at small scales.

Antokhin: Yes, this is correct. Still, I think it is worth trying to incorporate your (extrapolated to small scales) empirical correlations into existing atmosphere models.

Hillier: The spectrum of 10830 in WR 134 is an excellent example of a blend between $\mathrm{P} \gamma$ and HeI 10830. Other profiles in the Eenens paper probably provide a better example of unusual profiles.

Williams: I have examined the contribution of $\mathrm{P} \gamma$ and its helium analgues to the 10830 profile in WR 134 and they cannot account for the emission peak.

Pollock: What is the relation between supersonic turbulence and the strong shocks? Does the radiative instability thought responsible for the shocks also provide the driving mechanism for the turbulence?

Owocki: I believe what you refer to as "shocks" and "supersonic turbulence" should be thought of as the same thing. The line-driven instability is a good potential mechanism for generating this turbulence and maintaining it against the strong dissipation arising from its compressive nature. Recent calculations suggest that WR winds may indeed be subject to a moderately strong line-driven instability.

Vreux: Concerning the profile of HeI $\lambda 10830$ in WR 134, I would like to point out the following. It is true that most of the time P. Eenens and myself have observed the profile you have shown i.e. there is a small emission peak on the red side of the top of the HeI $\lambda 10830$ line. Nevertheless, I have one night, with a fair $\mathrm{S} / \mathrm{N}$, showing a double peaked profile, i.e. a small peak is also present on the blue side of the top of the HeI line. Then, this profile is reminiscent of some of the ones predicted by A. Underhill when she assumes that there is a ring around a star. But P. Eenens's suggestion most probably is also still possible. 